\title{
Article
}

\section{Comprehensive Evaluation of the GF-4 Satellite Image Quality from 2015 to 2020}

\author{
Wei Yi ${ }^{1, *}$, Yuhao Wang ${ }^{1}$, Yong Zeng ${ }^{1}$, Yaqin Wang ${ }^{2}$ and Jianfei $\mathrm{Xu}^{1}$ \\ 1 Department of Remote Sensing Satellite Operation, China Center for Resources Satellite Data and \\ Application, Beijing 100094, China; wangyuhao@spacechina.com (Y.W.); zengyong@spacechina.com (Y.Z.); \\ xujianfei@spacechina.com (J.X.) \\ 2 China Land Surveying and Planning Institute, Beijing 100035, China; wangyq.14b@igsnrr.ac.cn \\ * Correspondence: yiwei@spacechina.com
}

check for updates

Citation: Yi, W.; Wang, Y.; Zeng, Y.; Wang, Y.; Xu, J. Comprehensive Evaluation of the GF-4 Satellite Image Quality from 2015 to 2020. ISPRS Int.

J. Geo-Inf. 2021, 10, 406. https:// doi.org/10.3390/ijgi10060406

Academic Editors: Wolfgang Kainz and George P. Petropoulos

Received: 3 May 2021

Accepted: 8 June 2021

Published: 12 June 2021

Publisher's Note: MDPI stays neutral with regard to jurisdictional claims in published maps and institutional affiliations.

Copyright: (c) 2021 by the authors. Licensee MDPI, Basel, Switzerland. This article is an open access article distributed under the terms and conditions of the Creative Commons Attribution (CC BY) license (https:// creativecommons.org/licenses/by/ $4.0 /)$.

\begin{abstract}
GaoFen-4(GF-4) is the highest spatial resolution Earth observation satellite operating in geosynchronous orbit. Its fixed Earth observation location, rapid responsiveness, and wide observation range make it popular in disaster and emergency monitoring. To evaluate the GF-4 image quality in detail on a long-term basis, this study analyzes the image quality after the commissioning phase by focusing on ground sample distance (GSD) and geometric and radiometric quality. The theoretical calculation, geometric and radiometric measurements, and on-site experiments results show that (1) the GSD of the GF-4 image is $\sim 50 \mathrm{~m}$ at the nadir point and increases gradually with the distance away from the nadir point, (2) most external geometric errors are within the design requirements of $4 \mathrm{~km}$ despite some exceeding the limit, and the internal geometric errors are tested within 1 pixel, and (3) image sharpness is generally stable but varies with the atmosphere condition and imaging time, and the radiometric response gradually degrades at the rate of less than 5.5\% per year.
\end{abstract}

Keywords: GF-4; Earth observation satellite; geosynchronous orbit; assessment

\section{Introduction}

An Earth observation satellite is one of the most convenient and efficient means to obtain land surface information at a global or regional scale. Most satellites are operating in the repeat sun-synchronous orbit so they fly through the target place at the same local time within the scheduled time interval. Given the consistency of sun illumination conditions, data obtained on different days are comparable [1,2]. However, if data are needed instantly for a particular area, especially in locations where disasters occur, most satellites cannot meet the demand. A geosynchronous orbit satellite can greatly fill the gaps and obtain high temporary and spatial resolution $(\sim 50 \mathrm{~m})$ data at any time by adjusting the satellite pointing. Many countries have launched geosynchronous satellites, most of them with a spatial resolution greater than $250 \mathrm{~m}$. Table 1 shows the spatial resolution of geosynchronous satellites launched in recent years. China has recently implemented the High-Resolution Earth Observation System or the GaoFen plan and launched a series of satellites to meet the demands of Earth observation and its application in China [3]. The plan includes seven civil Earth observation satellites, one of which is GaoFen-4 (GF-4), which has the highest spatial resolution instrument in the world. 
Table 1. Information of the latest geosynchronous satellites.

\begin{tabular}{cccccc}
\hline Series & $\begin{array}{c}\text { Latest } \\
\text { Satellite }\end{array}$ & Sensor & $\begin{array}{c}\text { Spatial } \\
\text { Resolution/m }\end{array}$ & $\begin{array}{c}\text { Country or } \\
\text { Organization }\end{array}$ & Launch Year \\
\hline Meteosat & Meteosat-11 & SEVIRI & 500 & European Union & 2015 \\
Himawari & Himawari-9 & AHI & 1000 & Japan & 2016 \\
INSAT & INSAT-3DR & IMAGER & 1000 & India & 2016 \\
GEOS & GEOS-17 & ABI & 1000 & America & 2018 \\
COMS & Cheollian 2B & GOCI-2 & 250 & South Korea & 2020 \\
\hline
\end{tabular}

The GF-4 satellite was successfully launched at the Xichang Satellite Launch Center, China on 29 December 2015. It is the first geosynchronous orbit satellite in China and is equipped with a starring area array Complementary Metal Oxide Semiconductor (CMOS) camera that exceeds a $400 \mathrm{~km}$ wide swath. The camera can provide a $50 \mathrm{~m}$ visible and near infrared image and a $400 \mathrm{~m}$ mid-infrared image; moreover, the former has five bands in panchromatic, blue, green, red, and near-infrared wavelength [4-6]. The spectral bands are optional in satellite tasks. Table 2 shows the parameters of the image. The design life of the GF-4 is 8 years, and the satellite has been safely and stably operated by the China Center for Resources Satellite Data and Application (CRESDA) for more than 5 years. The daily schedule is coded into digital instructions and sent ahead to the satellite by 1 to 2 days. In case of a natural disaster or emergency, the instructions can also be temporarily altered in a few minutes. Data are freely available to the public, and the relevant information is accessible at http:/ /36.112.130.153:7777/DSSPlatform/productSearch.html (accessed on 20 March 2021), with the interface in Chinese.

Table 2. Parameters of the GF-4 image.

\begin{tabular}{|c|c|c|c|c|c|c|}
\hline Satellite & Image & Band & $\begin{array}{c}\text { Spectral } \\
\text { Range/ } / \mu \mathrm{m}\end{array}$ & $\begin{array}{c}\text { Spatial } \\
\text { Resolution/m }\end{array}$ & $\begin{array}{l}\text { Quantity } \\
\text { Level/bits }\end{array}$ & Width $/ \mathbf{k m}$ \\
\hline \multirow{6}{*}{ GF-4 } & \multirow{5}{*}{$\begin{array}{l}\text { Panchromatic and } \\
\text { Multispectral (PMS) }\end{array}$} & PAN & $0.45-0.90$ & \multirow{5}{*}{50} & \multirow{5}{*}{10} & \multirow{6}{*}{400} \\
\hline & & B1 & $0.45-0.52$ & & & \\
\hline & & B2 & $0.52-0.60$ & & & \\
\hline & & B3 & $0.63-0.69$ & & & \\
\hline & & B4 & $0.76-0.90$ & & & \\
\hline & Mid-infrared (IR) & B5 & $3.5-4.1$ & 400 & 12 & \\
\hline
\end{tabular}

The ground sample distance (GSD) is the first consideration in practice because it determines whether or not the targets can be identified in the image. Ground control points are essential when high geo-positioning accuracy images are required [6,7]. To examine the quantitative retrieval, radiometric and atmospheric corrections should be performed with calibration coefficients $[8,9]$. These coefficients can also be obtained from the CRESDA website. However, for a satellite with large-area capability such as the GF4 , the GSD varies considerably in different geolocations. Meanwhile, directly using the GSD is convenient if the geometric accuracy of the original image sufficiently matches the application requirements. The calibration coefficients of the previous year can also be used before those of the later year are released to the public if the radiometric response is stable. Hence, the stability and reliability of GF-4 images are of great importance for applications, a feature which is also the most concerning aspect for users.

Several studies have been conducted from one perspective to another $[4,10-13]$. Wang et al. analyzed the source of GF-4 geometric error and summarized the rule of error variation. The average external geometric accuracy was improved from $1750 \mathrm{~m}$ to $25 \mathrm{~m}$, and the average internal geometric accuracy was improved from 3.5 pixels to 0.4 pixels after geometric correction [4]. Wei et al. evaluated nine GF-4 images according 
to 630 GCPs in coastal zones and offshore areas and obtained a geolocation uncertainty of $1925 \pm 976 \mathrm{~m}$ [10]. Li et al. proposed a framework to refine RPC parameters, decreasing the RMSE of the georeferencing from $3894.4 \mathrm{~m}$ to $86.7 \mathrm{~m}$. These studies concentrated on the geometric quality of GF-4 images, while radiometric quality was rarely discussed in published literature. The commonly used method is to cross-calibrate the radiometric value of GF-4 images with a well-calibrated satellite as the reference sensor [13]. However, the image quality of the GF-4 satellite could vary because of the on-orbit life phases [14-17]. That quality can also be affected by different factors, including the modification of camera parameters and the motion and jitter of the platform. The evaluation results even vary with the data acquired on the same day $[5,18]$. The individual image quality cannot represent the images in the entire on-orbit lifetime. Therefore, long-term image quality is essential for users to make better use of the data. For this reason, this article aims to evaluate the GSD variation with geolocations according to theoretical calculation, and summarize the geometric and radiometric characteristics over the past 5 years from an analysis of a large volume of data acquired from the launch.

\section{Evaluation of the GSD}

The GSD is equivalent to the spatial resolution of an image most of the time. It is also consistent unless the satellite altitude is greatly adjusted. For example, the GSD decreases with increasing altitude, and vice versa. For the spatial resolution, all pixels in an image should be the same after orthorectification. The spatial resolution of the GF-4 released to the public is $50 \mathrm{~m}$ in the visible and near infrared wavelengths and $400 \mathrm{~m}$ in the mid-infrared wavelength. That resolution is only correct for the image acquired at the nadir point or the neighboring areas. Figure 1 shows the nadir point located at the longitude of 105.6 on the equator and the scope areas it can capture.

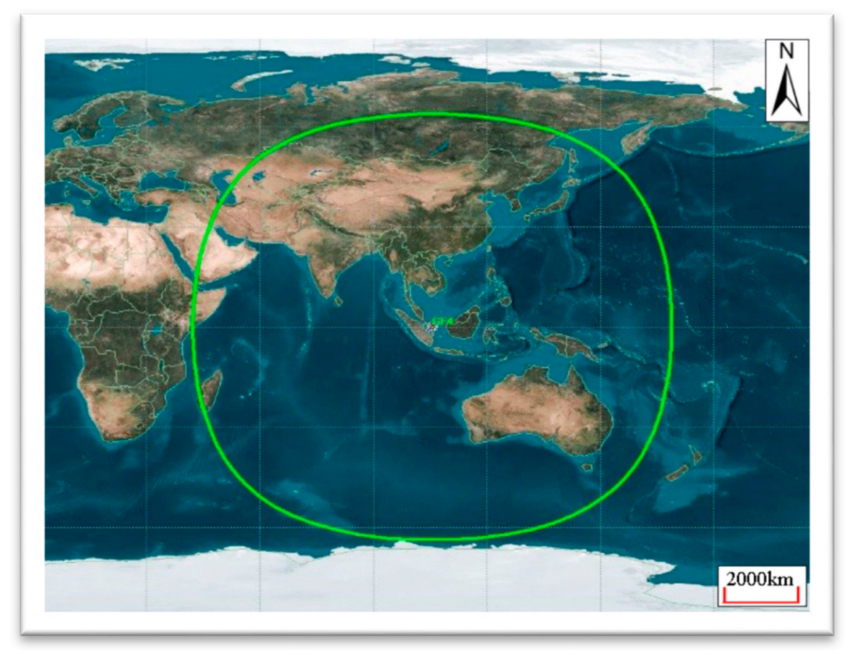

Figure 1. Area scopes that the GF-4 satellite can capture.

The GSD is determined by the instant field of view (IFOV) of the detector, the Earth curvature, and the satellite altitude. Figure $2 \mathrm{a}$ is the schematic diagram of the GSD at the nadir point. $\mathrm{S}$ is the position of the satellite, $\mathrm{N}$ is the nadir point, $\mathrm{R}$ and $\mathrm{L}$ are the edges of the ground track of a detector, and $\mathrm{O}$ is the center of the Earth. According to the principles of the triangle function, the geocentric angle corresponding to the half of GSD can be calculated as

$$
\beta=\arcsin \frac{(\operatorname{Re}+H) \sin \theta}{\operatorname{Re}}-\theta
$$

where $\beta$ is the geocentric angle, Re is the radius of the Earth, $H$ is the satellite altitude, and $\theta$ is the half IFOV of a detector. 
Then, the GSD can be calculated with a geocentric angle as

$$
G S D_{\text {nadi } r}=2 \operatorname{Re} \beta=2 \operatorname{Re}\left[\arcsin \frac{(\operatorname{Re}+H) \sin \theta}{\operatorname{Re}}-\theta\right]
$$

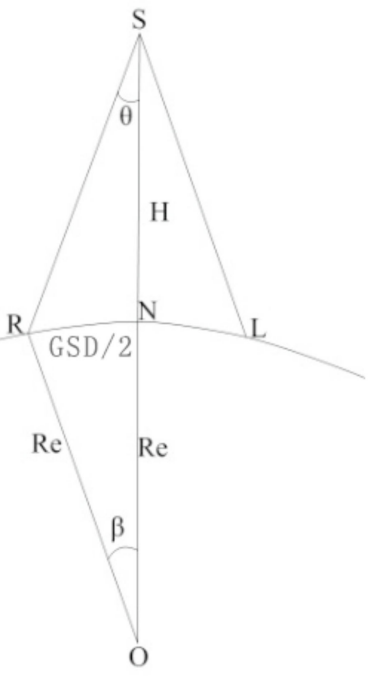

(a)

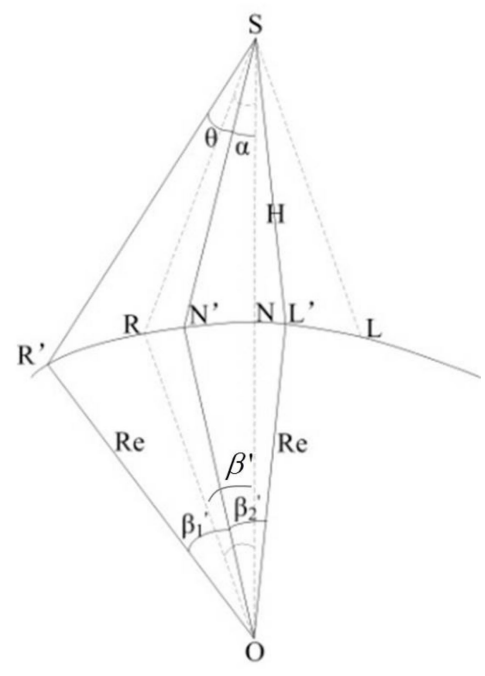

(b)

Figure 2. The schematic diagram of the GSD. (a) the nadir point; (b) off the nadir point.

According to Formula (2) and the designed IFOV value, the GSD is $48.62 \mathrm{~m}$ at the nadir point, an outcome which is better than the released spatial resolution.

The GSD along the imaging direction (hereafter referred to as the along-track direction) equals that across the imaging direction (hereafter referred to as the across-track direction) at the nadir point, whereas they are inconsistent at other locations. Figure $2 \mathrm{~b}$ shows the schematic diagram of the GSD in the along-track direction at the off-nadir point. The corresponding GSD can be calculated as

$$
\begin{gathered}
G S D_{\text {al ong }}=\operatorname{Re}\left(\beta_{1}^{\prime}+\beta_{2}^{\prime}\right) \\
=\operatorname{Re}\left(\arcsin \frac{(\operatorname{Re}+H) \sin (\theta+\alpha)}{\operatorname{Re}}+\arcsin \frac{(\operatorname{Re}+H) \sin (\theta-\alpha)}{\operatorname{Re}}-2 \theta\right)
\end{gathered}
$$

where

$$
\left\{\begin{array}{l}
\beta_{1}^{\prime}=\angle R^{\prime} O N-\angle N^{\prime} O N=\arcsin \frac{(\operatorname{Re}+H) \sin (\theta+\alpha)}{\operatorname{Re}(\theta)}-\arcsin \frac{(\operatorname{Re}+H) \sin \alpha}{\operatorname{Re} \sin \alpha}-\theta \\
\beta_{2}^{\prime}=\angle N^{\prime} O N+\angle L^{\prime} O N=\arcsin \frac{(\operatorname{Re}+H) \sin (\theta-\alpha)}{\operatorname{Re}}+\arcsin \frac{(\operatorname{Re}+H) \sin }{\operatorname{Re}}-\theta
\end{array}\right.
$$

for which $\alpha$ is the viewing angle of the satellite.

The GSD across the imaging direction is much simpler and can be calculated as

$$
G S D_{\text {acr oss }}=2 \operatorname{Re}\left[\arcsin \frac{\left(\operatorname{Re}+H^{\prime}\right) \sin \theta}{\operatorname{Re}}-\theta\right]
$$

where

$$
H^{\prime}=\operatorname{Re} \frac{\sin \beta^{\prime}}{\sin \alpha}
$$

In the two-dimensional orthorectified image, the resolutions of the sample and line in a pixel should be the same. To represent the resolution of a pixel, a geometric GSD is needed and is represented as

$$
G S D_{\text {geo }}=\sqrt{G S D_{\text {along }} \times G S D_{\text {across }}}
$$


Figure 3 shows the variation of the GSD with the latitude starting from the equator, and with several longitudes as examples with an interval of 10 degrees. If the interval is -10 degrees or if latitudes are taken as examples to show the variation of the GSD with the longitude, the result is the same due to the symmetry of the sphere. The latitude and longitude ranges do not correspond to the limits that the GF- 4 can capture, but the image distortion beyond the scope is enhanced. Thus, this work shows only the latitude from 0 to 60 and the longitude from 0 to 40 off the nadir point.

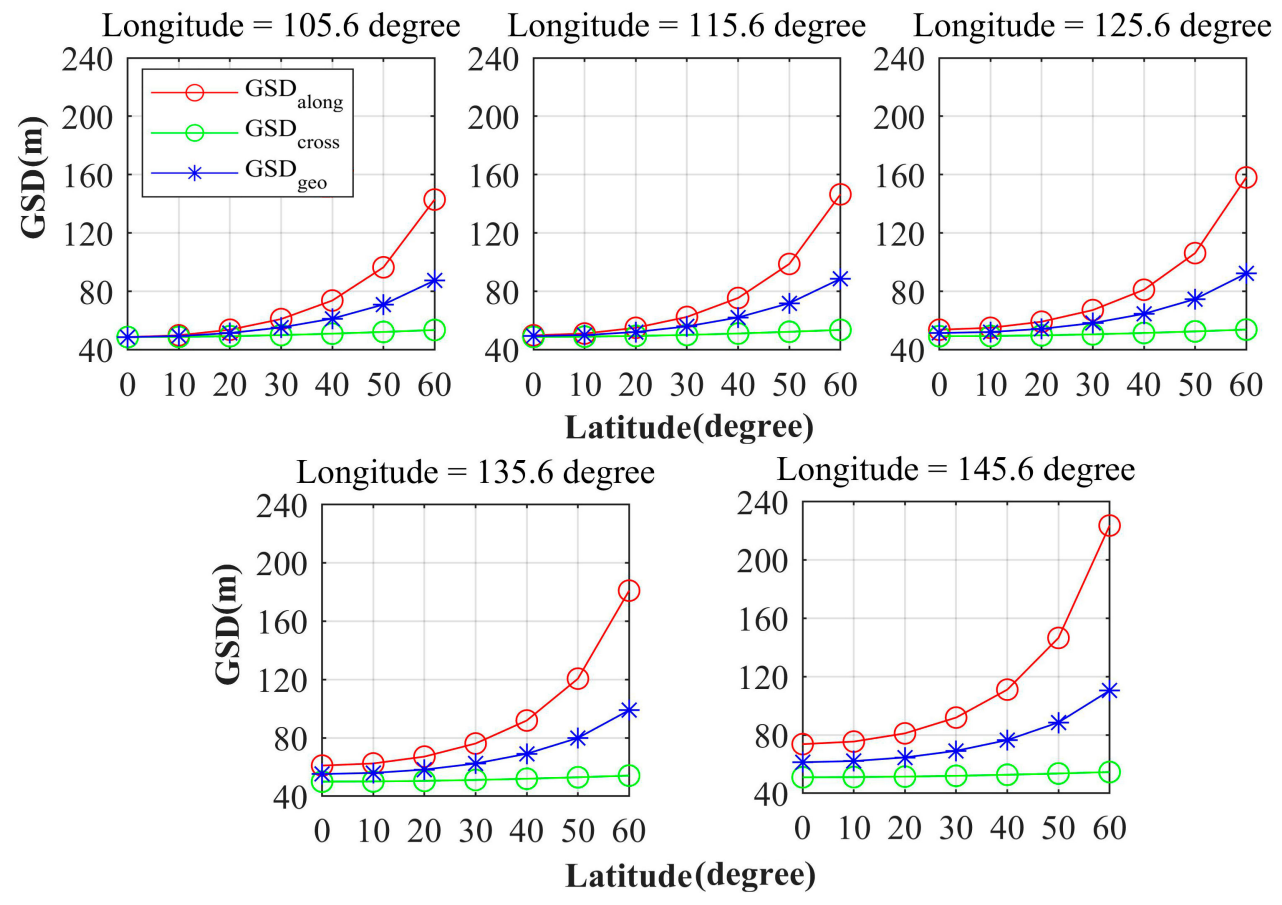

Figure 3. The variation of GSD with latitude and longitude.

The GSD in the along-track and across-track directions notably differ. As the distance increases from the nadir, the GSD in the across-track direction varies little, compared with that in the along-track direction. When the latitude and longitude are 60 and 40 degrees off the nadir point, respectively, the variation in the across-track direction is only 1.12 times that of the nadir point, while it is more than 4.6 times in the along-track direction. The GSD variance in the longitude of 105.6 reveals the characteristics in one dimension. At the nadir point, GSDs in the along-track direction and the across-track direction are consistent, but the gap becomes gradually larger with the increase in latitude. At other points with a different longitude, GSDs in the along-track direction and the across-track direction are different even on the equator.

The variation of the GSD also leads to the change of swath and coverage, which are smallest at the nadir point and largest near the limit of GF-4 capturing capability. This change is not completely proportional to the GSD due to its difference among pixels in the same image. Regarding the four corners of the image, the resample size of a corner far away from the nadir point is much larger than that near the nadir point, thereby causing the shape of the image to be a distorted diamond. Figure 4 shows the shape of the original Level 1A image and its orthorectification image in the northeast of China. 


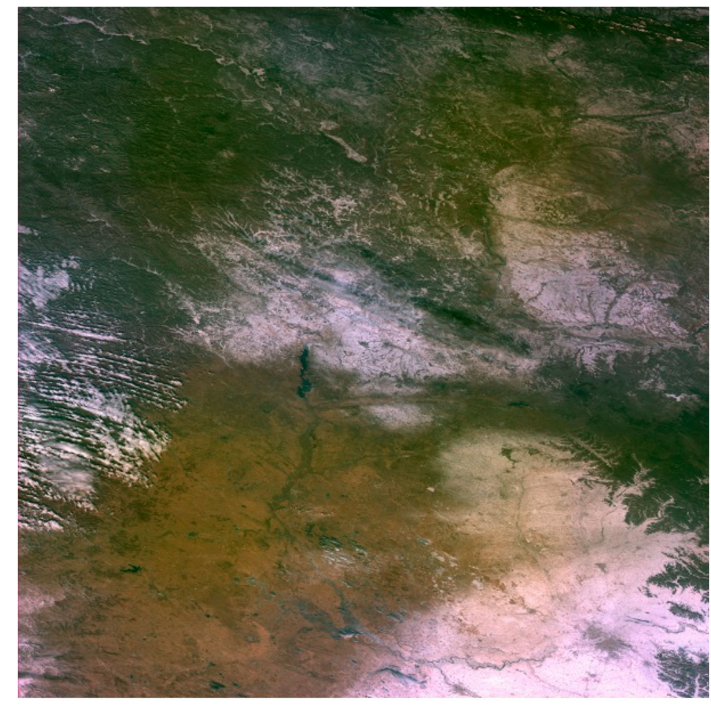

(a)

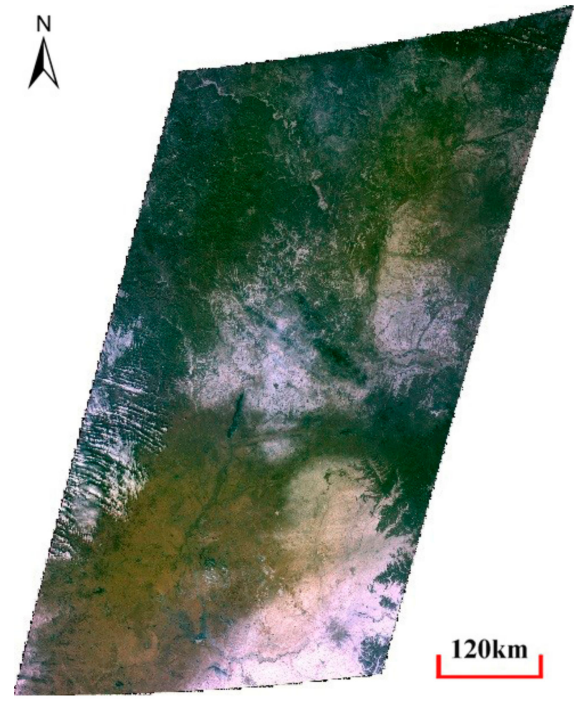

(b)

Figure 4. Shape of the original Level 1A image (a) and its orthorectification image (b) in the northeast of China.

\section{Evaluation of the Geometric Quality of the Image}

Geometric quality can be divided into external and internal geometric accuracies. External geometric accuracy refers to the deviation between the coordinate positions of checkpoints and their actual positions, also known as absolute positioning accuracy. Internal geometric accuracy pertains to the deformation of an image which can be regarded as the consistency among pixels of the entire image or the relative positioning accuracy.

\subsection{External Geometric Accuracy}

External geometric accuracy is evaluated with level-1A data, and two additional data are needed. One is Digital Elevation Model (DEM) data extracted from STRM data [19], released by the U.S. Geological Survey (USGS), and with a spatial resolution of $30 \mathrm{~m}$. The resolution is comparable with that of GF-4 data. The other is accurate geo-positioning reference data. Landsat is used here, with a positioning accuracy exceeding $15 \mathrm{~m}$ [20]. This value is precise enough to test the external geometric accuracy of GF-4 data because the root mean square error (RMSE) of the design requirement is $4 \mathrm{~km}$.

The data selection principle involves covering extensive areas under different terrain conditions and at least one scene per week to form a long-term dataset. As data before May 2016 are under the commissioning phase, they are restrictively released to the public and are thus excluded from this work. The data should be of normal quality and cloud-free so as to facilitate checkpoint selection. The test processes of each scene are as follows:

(1) A total of 20-30 checkpoints that were evenly distributed in the image were selected. Given the maturity of current image registration technology, this step can be done with auto-registration software.

(2) Coordinate inverse calculation was conducted with reference data, RPC file, and DEM data to convert the positions of checkpoints in the reference image from the ground space to the image space, as represented by the sample and line.

(3) The differences of the samples and lines between the reference image and the corresponding GF-4 image were calculated as

$$
\begin{aligned}
& X_{i}^{j}=x_{i}^{j}-x_{i 0}^{j} \\
& Y_{i}^{j}=y_{i}^{j}-y_{i 0}^{j}
\end{aligned}
$$

where $X_{i}^{j}$ and $Y_{i}^{j}$ are the sample and line errors of the ith checkpoint in scene $j$, respectively; $x_{i}^{j}$ and $y_{i}^{j}$ are the sample and line of the ith checkpoint in scene $j$, respectively; $x_{i 0}^{j}$ and $y_{i 0}^{j}$ 
are the corresponding sample and line of the ith checkpoint converted from the ground space to the image space.

(4) The mean error was calculated as the external geometric accuracy of the scene as

$$
\begin{gathered}
\mu_{x}^{j}=\frac{1}{n} \sum_{i=1}^{n}\left(x_{i}^{j}-x_{i 0}^{j}\right) \\
\mu_{y}^{j}=\frac{1}{n} \sum_{i=1}^{n}\left(y_{i}^{j}-y_{i 0}^{j}\right) \\
\mu^{i}=\sqrt{\left(\mu_{x}^{j}\right)^{2}+\left(\mu_{y}^{j}\right)^{2}}
\end{gathered}
$$

where $\mu_{x}^{j}$ and $\mu_{y}^{j}$ are the mean sample and line error of the scene $j$, respectively, and $\mu^{i}$ is the external geometric accuracy of the scene $j$. The mean sample and line error represent the errors in the along-track and across-track directions, respectively.

Figure 5 shows the error direction of each scene. From the directionality of the external geometric accuracy, errors are distributed randomly in four quadrants during the on-orbit phase, and the average error is approximately zero in either the along-track or cross-track directions. Thus, no systematic error occurs, otherwise the values would be biased in a certain direction. The RMSE indicates that $68.3 \%$ of the data should meet the design requirement according to the probability distribution. The external geometric accuracy is in accord with the expectation as the RMSE is $3.89 \mathrm{~km}$. At the same time, CE90 is also given as $5.67 \mathrm{~km}$. Given that a few individual outliers were omitted in this study, a low probability exists that the conclusion may not apply to all data. Although the external geometric accuracy matches the design requirement, note that not all images have the same geometric accuracy.

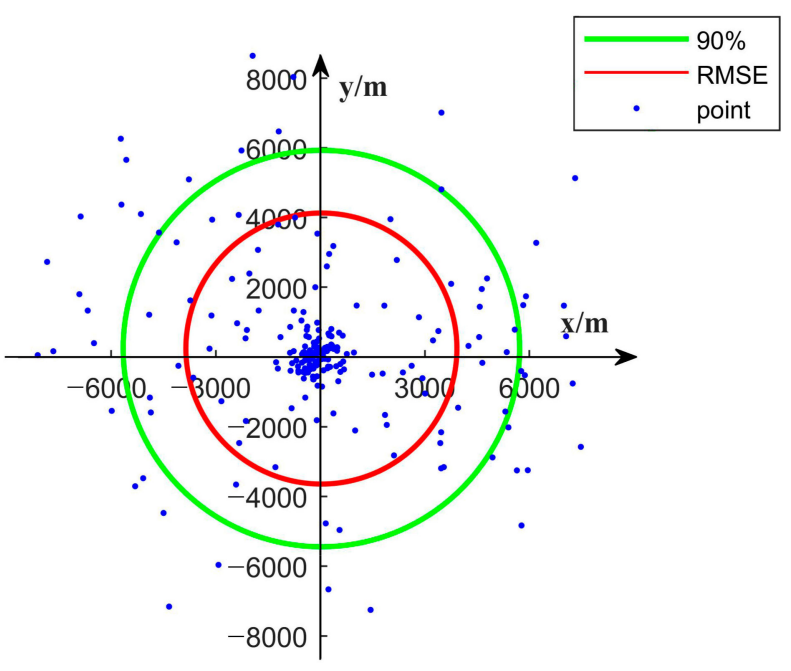

Figure 5. External geometric accuracies of selected GF-4 images.

The average external geometric accuracy of each month represents the absolute positioning accuracy variance with time (Figure 6). The trend of average external geometric accuracy gradually increased at certain times, suddenly decreased at some points, and then increased as usual. This situation arises because the quality control group detected the deviation of external geometric accuracy when it started to exceed the limit and updated the parameters of the rigorous geometric model in the ground data processing system so as to improve the data quality. At the same time, the trend is not completely linear and occasionally has some randomness, an occurrence which is related to the motion, jitter, and measurement accuracy of the platform. 


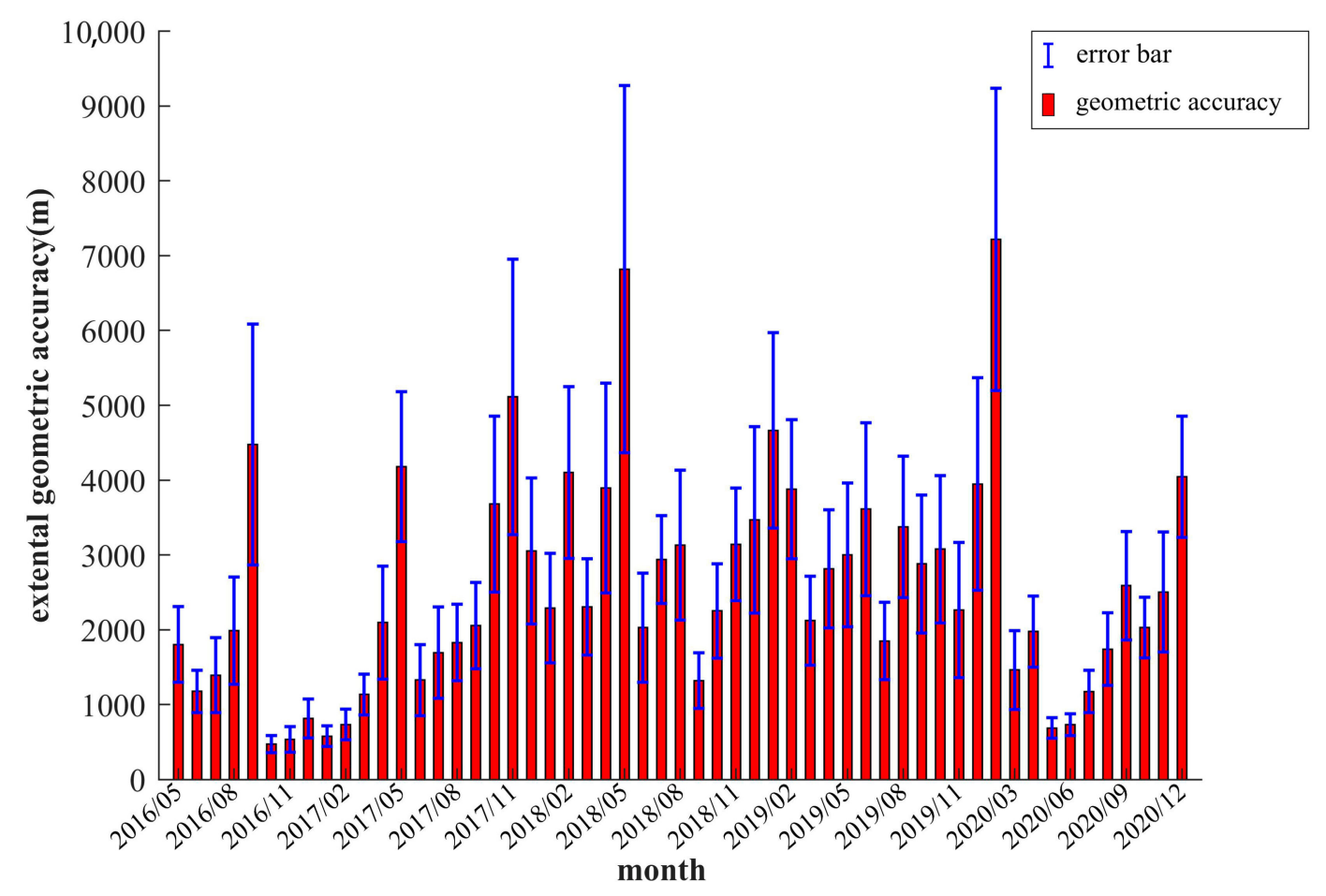

Figure 6. Variation of the external geometric accuracy with time.

\subsection{Internal Geometric Accuracy}

Internal geometric accuracy generally draws less attention than its external counterpart because its value is so small that it can be ignored. For the push broom image of a linear array camera, the internal geometric accuracy is considered as the RMSE of the residuals of checkpoints after removing the external geometric accuracy. However, for an area array imaging camera such as the GF-4, images are mainly acquired through the rotation of the satellite. Therefore, in addition to the external geometric accuracy, the rotation error should also be removed because it can be corrected by altering the rigorous geometric model. These two errors can be regarded as system errors. After removing them, the RMSE of residuals reflects the internal geometric accuracy.

Internal geometric accuracy was measured and adjusted in the laboratory before the launch. The influence of temperature variance from the ground to space means that the shape of the detector may change slightly, thereby resulting in image distortion. If the internal geometric accuracy is less than 1 pixel, then the data application will be unaffected. As the space environment in space is much more stable than that on the ground, the variation would be minimal in the later phase if the distortion occurs in the early phase after the launch and was adjusted in the data processing flow. Therefore, only two images were selected for evaluation in this study. The date of one scene is shortly after the launch, and that of the other is at the end of December 2020. Figure 7 shows that the relative errors and direction of the checkpoints in the two images before and after the removal of system errors. Note that the length of the arrows serves only to enhance the visual effect and demonstrates the relative value of errors in an image and not the absolute value. Thus, no comparability occurs between images before and after the removal of external geometric accuracy.

The errors are directional before systematic correction and randomly distributed after correction. At the same time, the absolute values of the internal geometric accuracy in the early phase are 0.67 and 0.57 pixels in the along-track and across-track directions, respectively, and that of the latest image are 0.54 and 0.65 pixels. All these values meet the requirement of less than 1 pixel. 


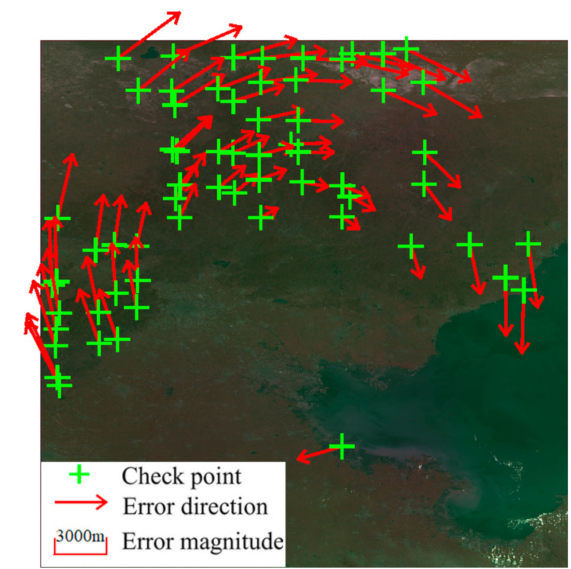

(a)

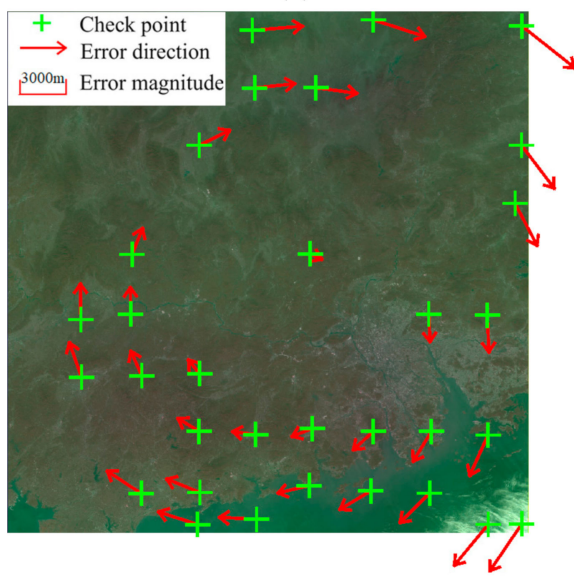

(c)

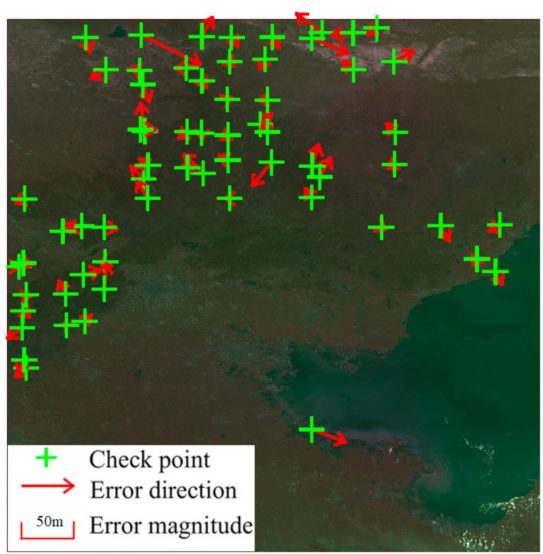

(b)

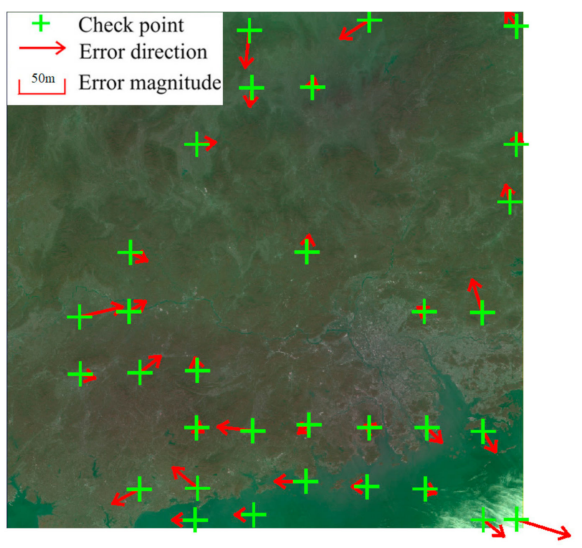

(d)

Figure 7. The relative errors and their directions of checkpoints. Errors of an image (a) on 28 August 2016 before removing the external geometric accuracy; (b) on 28 August 2016 after removing the external geometric accuracy; (c) on 21 December 2020 before removing the external geometric accuracy; (d) on 21 December 2020 after removing the external geometric accuracy.

\section{Evaluation of the Radiometric Quality of an Image}

Radiometric quality is satisfactory when it performs well in relation to image sharpness and radiometric response. The modulation transfer function (MTF) and signal-noise ratio (SNR) are commonly used to evaluate image radiometric quality [21,22], especially for high-resolution images such as IKONOS or QuickBird. However, obtaining a large number of data containing "knife-edge" targets or uniform areas is difficult because of the comparatively low resolution of GF-4 data. Therefore, a power spectrum is instead employed to evaluate image sharpness and reflects the displacement of the focal plane [23]. Radiometric calibration was conducted in the laboratory before the launch. Given the long transfer length along the atmosphere and some other factors, the calibration coefficients should vary from those obtained in the laboratory. The calibration team of CRESDA routinely updates and releases the coefficients to the public after conducting annual on-site experiments.

\subsection{Image Sharpness}

The power spectrum transforms an image from the spatial to the frequency domain so as to evaluate whether the focal plane of the optical system is displaced or not. The power spectrum of an image is defined as:

$$
|F(\mathrm{u}, \mathrm{v})|^{2}=F(\mathrm{u}, \mathrm{v}) * F^{*}(\mathrm{u}, \mathrm{v})
$$


where $F(\mathrm{u}, \mathrm{v})$ is the Fourier transform of image $\mathrm{f}(\mathrm{x}, \mathrm{y}), F^{*}(\mathrm{u}, \mathrm{v})$ is the conjugate function of $F(\mathrm{u}, \mathrm{v}), \mathrm{u}$ is the horizontal frequency, and $\mathrm{v}$ is the vertical frequency.

The power spectrums of two-dimensional images as calculated by the above formula are not readily comparable to one another. Therefore, to show the trend of the power spectrum, Formula (11) is typically used to convert a two-dimensional power spectrum into a one-dimensional counterpart.

$$
P(\rho)=\frac{1}{n_{\rho}} \sum_{\theta=-180^{\circ}}^{\theta=+180^{\circ}}|F(\rho, \theta)|^{2}
$$

where $F(\rho, \theta)$ is the polar formulation of $F(\mathrm{u}, \mathrm{v}), \rho$ is a radius, $\theta$ is an angle, and $n_{\rho}$ is the number of points on the ring with the radius of $\rho$.

To remove the effect of image size, the power spectrum can be transformed as

$$
P(\rho)=\frac{1}{M_{\rho} \times N_{\rho}} \sum_{\theta=-180^{\circ}}^{\theta=+180^{\circ}}|F(\rho, \theta)|^{2}
$$

where $M_{\rho}$ is the samples of the image and $N_{\rho}$ is the lines of the image.

To compare a series of images, a variable is used to represent the power spectrum and is defined as

$$
I=\int_{\rho=0}^{0.5} P(\rho) \mathrm{d} \rho
$$

The image consisting of various land covers is more likely to be detected out of focus, so images of cities and their neighboring areas are optimal for study. To ensure the reliability of the evaluation, a long-term time series of images covering Chifeng and Zhengzhou (which are located in the north and middle of China, respectively) are selected as contrast experiments. The two cities have high acquired ratios of cloud-free images which can provide more data for the evaluation. A total of 208 scenes (82 in Zhengzhou and 157 in Chifeng) from May 2016 to July 2020 were available after strict selection and filtration.

The scatter plot of the power spectrum and the day shows that simply comparing the power spectrums of two images fails to truly reflect the change of image sharpness, as shown in Figure 8. The focus of the optical system always slowly displaces after the commissioning phase and should not have a sharp change in a short period according to engineering experience. The imaging time of these images notably vary from the morning to the afternoon. Hence, the difference between two images is caused not only by defocusing, but also by the atmosphere condition, season variation, and acquisition time. The mixed factors complicate the comparison of any two images, but we can also find some characteristics from the statistical analysis of extensive data. First, the variances of the power spectrums of the two study areas almost locate in the same value range (a minimum above 50 and a maximum of approximately 200) and almost no abnormal value occurs. From this viewpoint, the change of image sharpness is very stable. The average power spectrum is also concentrated at around 135. Second, the trend analysis shows that the power spectrums of the two study areas almost coincide. The intercepts of Chifeng and Zhengzhou are 135.11 and 134.16, respectively, and the slope is negative around zero. If the statistic scale of the slope is prolonged to a year, then the value is less than $0.1 \%$ annually. This outcome indicates that the image sharpness degrades slightly because of defocusing and that the degree is low or even negligible. Note that some minor differences arise in the trend analysis of the slopes and intercepts between Chifeng and Zhengzhou. The main reason for this result is that the amount of cloud-free data is insufficient. The amount of data in Zhengzhou in the early years is quite limited. In theory, if a large volume of data is accumulated in the upcoming years, then the gap should be minimal. 


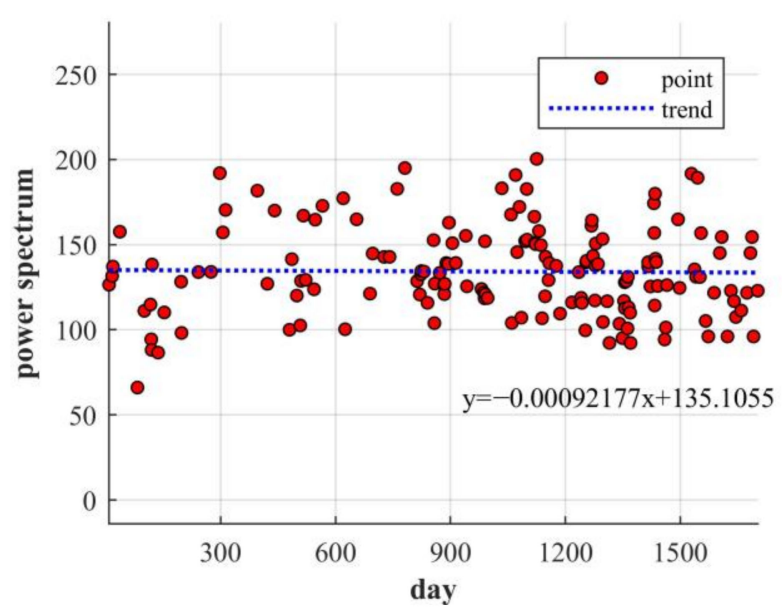

(a)

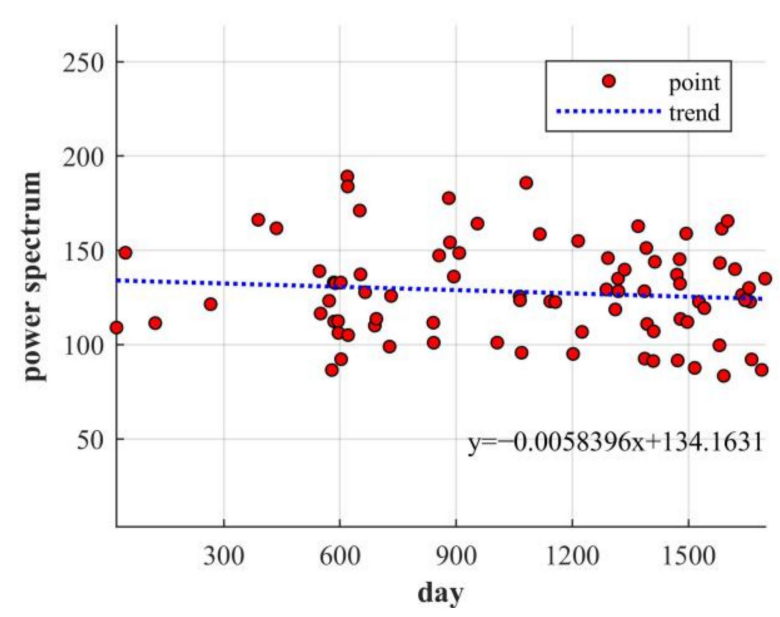

(b)

Figure 8. Trend of the power spectrum with time in Chifeng (a) and Zhenzhou (b).

\subsection{Radiometric Response}

To monitor radiometric response characteristics, ground-based observation experiments are conducted annually at the Dunhuang test site. The site $\left(40^{\circ} 5^{\prime} 32.80^{\prime \prime} \mathrm{N}, 94^{\circ} 23^{\prime} 35.78^{\prime \prime} \mathrm{E}\right)$ is located on the eastern edge of the Kumutage Penniform Desert (which is part of the Gobi Desert in the northwestern area of China) and lies approximately $35 \mathrm{~km}$ to the west of Dunhuang City, Gansu Province. The land surface covered by cemented gravels is optimal for the radiometric experiments of satellite sensors [24,25].

The reflectance-based method synchronously measures the surface reflectance, atmospheric data, and other parameters within 30 minutes before and after the flight over the test site [26]. Ground reflectance is measured with a spectroradiometer device transported across the entire site. The radiance of a certain band at the sensor is calculated by the $6 \mathrm{~S}$ radiative transfer model [27], the input with spectral response function, the solar and satellite angle, and the atmospheric data collected at the same time as the ground reflectance measurements. The calibration coefficient is then calculated according to the grey level of image and radiance as

$$
G=\frac{R_{a}-B}{D N}
$$

where $G$ is the gain of the radiometric response, $B$ is the bias of the radiometric response, and both are called calibration coefficients. $R_{a}$ is the radiance of a certain band at the sensor, and $D N$ is the corresponding grey level of an image. $B$ is assumed to be stable over these years and set to be zero in the released version. Given the difference in the integral level settings of a sensor, the ground tests and corresponding images should include as many possibilities as possible. Five commonly used settings are shown in Table 3, including the $G$ of visible and near-infrared bands, and Figure 9 shows the variation of G during 2016 to 2020 . These details are available on http:/ / www.cresda.com/CN/Downloads/dbcs/index.shtml (accessed on 20 March 2021).

The annual average relative variation is used to evaluate the stability of the radiometric response as

$$
\begin{gathered}
V_{i}=\frac{G_{i}-A v e_{i}}{A v e_{i}} \times 100 \% \\
V=\frac{\sum_{i=0}^{N} V_{i}}{N}
\end{gathered}
$$

where $V_{i}$ is the variance of the calibration coefficient in the ith year, $A v e_{i}$ is the average value of the calibration coefficient of $G$, and $V$ is the annual average relative variation. 
Table 3. The calibration coefficients of each band with different integral parameters from 2016 to 2020 .

\begin{tabular}{|c|c|c|c|c|c|c|}
\hline Integral Level & Band & 2016 & 2017 & 2018 & 2019 & 2020 \\
\hline \multirow{5}{*}{ PMS (2-6-4-6-6) } & PAN & 0.5215 & 0.5061 & 0.4933 & 0.5395 & 0.5329 \\
\hline & B1 & 0.94 & 0.9175 & 1.0904 & 1.0028 & 0.9767 \\
\hline & B2 & 0.9885 & 0.9691 & 1.1484 & 1.0418 & 1.0278 \\
\hline & B3 & 0.7847 & 0.7715 & 0.8558 & 0.8017 & 0.809 \\
\hline & B4 & 0.5641 & 0.5512 & 0.6046 & 0.5655 & 0.5738 \\
\hline \multirow{5}{*}{ PMS (4-16-12-16-16) } & PAN & 0.31 & 0.3151 & 0.3043 & 0.3327 & 0.3293 \\
\hline & B1 & 0.3484 & 0.3511 & 0.4175 & 0.3803 & 0.3728 \\
\hline & B2 & 0.3448 & 0.3641 & 0.4244 & 0.3863 & 0.3833 \\
\hline & B3 & 0.3095 & 0.3207 & 0.3505 & 0.3299 & 0.331 \\
\hline & $\mathrm{B} 4$ & 0.2257 & 0.2314 & 0.2494 & 0.2343 & 0.2363 \\
\hline \multirow{5}{*}{ PMS (6-20-16-20-20) } & PAN & 0.1683 & 0.1647 & 0.159 & 0.1752 & 0.1733 \\
\hline & B1 & 0.3263 & 0.3286 & 0.3833 & 0.3531 & 0.349 \\
\hline & B2 & 0.2472 & 0.2578 & 0.2998 & 0.275 & 0.2719 \\
\hline & B3 & 0.2806 & 0.2858 & 0.3109 & 0.2946 & 0.2988 \\
\hline & $\mathrm{B} 4$ & 0.1997 & 0.1999 & 0.215 & 0.2038 & 0.2082 \\
\hline \multirow{5}{*}{ PMS (6-40-30-40-40) } & PAN & 0.1678 & 0.1647 & 0.1577 & 0.1735 & 0.1725 \\
\hline & B1 & 0.1252 & 0.1315 & 0.1468 & 0.1375 & 0.1395 \\
\hline & B2 & 0.1226 & 0.1239 & 0.1427 & 0.1308 & 0.1312 \\
\hline & B3 & 0.1102 & 0.1154 & 0.1205 & 0.1171 & 0.1203 \\
\hline & $\mathrm{B} 4$ & 0.0796 & 0.0802 & 0.0858 & 0.0818 & 0.083 \\
\hline \multirow{5}{*}{ PMS (8-30-20-30-30) } & PAN & 0.1235 & 0.1221 & 0.116 & 0.1288 & 0.1266 \\
\hline & B1 & 0.1784 & 0.1749 & 0.2053 & 0.1887 & 0.1858 \\
\hline & B2 & 0.1878 & 0.1903 & 0.2209 & 0.203 & 0.2013 \\
\hline & B3 & 0.1515 & 0.1532 & 0.1664 & 0.1569 & 0.158 \\
\hline & B4 & 0.108 & 0.1073 & 0.115 & 0.1084 & 0.1087 \\
\hline
\end{tabular}

The integral level parameter is represented in the order of wavelength band PAN, B1, $\mathrm{B} 2, \mathrm{~B} 3$ and B4 (PAN-B1-B2-B3-B4). The most stable performance occurs under the integral level parameter (8-30-20-30-30). The annual average relative variation has the lowest value at $3.29 \%$, and the corresponding values of panchromatic, B1, B2, B3, and B4 are $2.82 \%$, $4.45 \%, 4.63 \%, 2.54 \%$ and $2.02 \%$, respectively. The maximum value with the parameter (4-16-12-16-16) is $3.95 \%$, and the corresponding values of each band are $3.20 \%, 5.32 \%, 5.49 \%$, $3.22 \%$ and $2.52 \%$. The stability of each band also varies with different integration level parameters. B4 seems to be the most stable band, for which the average annual relative variation is less than $3 \%$, followed by B3 and the panchromatic band. The uncertainty in the experiment process is greater than the variance among different integral level parameters or bands, and the maximum variance is only $5.5 \%$. Thus, the changing rate is acceptable. Many uncertainty factors can affect the accuracy, such as the operating specificity of the spectroradiometer, the non-Lambertian characteristics, the uniformity of the test site, and the change of the solar zenith angle during the measurement. 

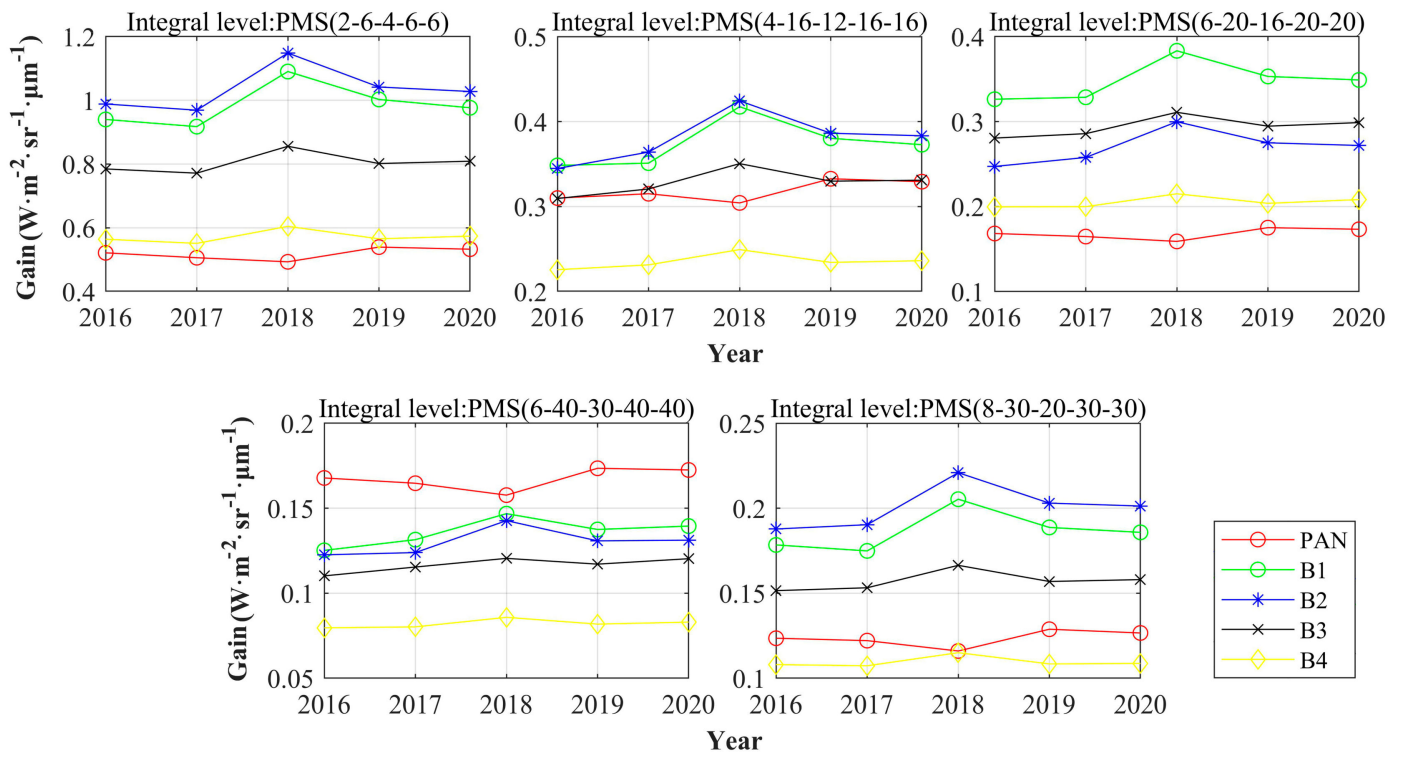

Figure 9. The calibration coefficients from 2016 to 2020.

\section{Discussion}

In general, the image quality evaluation of a remote sensing satellite includes geometric and radiometric quality [28]. Evaluation methods are common when it comes to geometric quality, i.e., comparing the target image with high-precision reference map by image registration technology. The external geometric accuracy of well-known geosynchronous orbit satellites such as GEOS-8, Meteosat- 1 and INSAT-3D are measured by the above methods, and the accuracies are 4 to $6 \mathrm{~km}$ [29], 4 to $5 \mathrm{~km}$ [30] and 10 to $20 \mathrm{~km} \mathrm{[28],}$ respectively. From the results in this paper, the geometric accuracy of GF-4 images is comparable with those of most geosynchronous orbit satellites, which shows the reliability of the method and the results. Surprisingly, the geometric accuracy of Himawari-8 image is close to 1 pixel. Note that the data are supposed to be geometrically corrected and then released to the public. Radiometric evaluation methods also vary with different satellites. The commonly used technique is SNR and MTF, especially for high-resolution images. Given the comparatively low resolution of GF-4 images, data containing "knife-edge" targets or uniform areas are difficult to obtain. Therefore, SNR and MTF are not used and power spectrum is instead employed to evaluate image sharpness. The results reflect the stability of the image sharpness and show no displacement of the focal plane. Moreover, radiometric response is a key factor of image quality. The radiometric coefficients of most satellites such as Landsat, Terra MODIS and EO-1 Hyperion [31,32] have been monitored for many years, which shows that this method is well recognized. The cameras have degraded in radiometric response by $10 \%$ since the launch of the EOS/Terra spacecraft [32], and the degradation rate is greater than that of GF-4. GSD is also a frequently applied image quality index for most satellites, and it varies considerably with geolocations [22] Thus, the analysis of the GSD variation is useful for users before they consider GF-4 images as an application data source. Given that the Earth's curvature and satellite altitude are constant, and we can deduce the IFOV according to the GSD at the nadir point, so the image GSD variation with geolocations is evaluated by theoretical value instead of measuring it in the image, which can also avoid the measuring errors in the actual images.

\section{Conclusions}

In this study, the evaluation of the quality of GF-4 satellite images is conducted from three aspects: GSD, geometric quality, and radiometric quality. Theoretical description and data analysis reveal the following conclusions.

The spatial resolution released to the public with $50 \mathrm{~m}$ in panchromatic and multispectral bands and $400 \mathrm{~m}$ in mid-infrared is only true under the condition that the image 
is acquired at nadir point or at the neighboring areas. For other regions of interest, the GSD increases gradually with the distance away from the nadir point. If the orthorectification is implemented with the level 1A image, the spatial resolution parameters should be determined by the longitude and latitude.

The geometric quality is evaluated from the external and internal geometric accuracies. The external geometric accuracy error directions evaluated on extensive images are inconsistent, so the judgment can be made that no systematic error occurs. Most of the errors are also within the design requirements of $4 \mathrm{~km}$. The internal geometric accuracy can be evaluated from the RMSE of the residual errors of the checkpoints after removing the systematic errors. The images acquired in the early and latest phases show that the accuracy is less than 1 pixel and meets the design requirements.

The radiometric quality of GF-4 images is evaluated in terms of image sharpness and radiometric response. The long-term monitoring results reveal that the variances of the power spectrum locate in a certain value range and indicates that the change of image sharpness is stable. However, the absolute value of one scene may differ from another because of the inconsistent imaging time and season or other affecting factors. The radiometric response is evaluated by the radiometric calibration coefficients according to annual on-site experiments. Moreover, the radiometric response of different bands and integral level parameters perform well with little degradation.

Author Contributions: Conceptualization, writing-review and editing, supervision, project administration, Wei Yi and Yuhao Wang; methodology, validation, formal analysis, Yong Zeng; investigation, data curation, visualization, Yaqing Wang; writing_original draft preparation, Jianfei Xu. All authors have read and agreed to the published version of the manuscript.

Funding: This work was funded by the Qianxuesen Innovation Foundation of China Aerospace Science and Technology Corporation (2020) and the National Natural Science Foundation of China (71771024).

Institutional Review Board Statement: Ethical review and approval were waived for this study, due to the data being sourced from an open platform.

Informed Consent Statement: Not applicable.

Data Availability Statement: Publicly available datasets were analyzed in this study. This data can be found here: (1) images were provided by official website of China Center for Resources Satellite Data and Application: http:/ /36.112.130.153:7777/DSSPlatform/productSearch.html (accessed on 20 March 2021). (2) The calibration coefficients were also provided by official website of China Center for Resources Satellite Data and Application: http:/ / www.cresda.com/CN/Downloads/dbcs/index. shtml (accessed on 20 March 2021).

Acknowledgments: The authors would like to thank the reviewers for their professional opinions and the hard work of the editorial department, which greatly promoted the improvement of the quality of the article. The authors would like to thank China Center for Resources Satellite Data and Application for providing us with data support.

Conflicts of Interest: The authors declare no conflict of interest.

\section{References}

1. He, Y.; Xu, M.; Jia, X.; Armellin, R. High-precision repeat-groundtrack orbit design and maintenance for Earth observation missions. Celest. Mech. Dyn. Astron. 2017, 128, 275-294. [CrossRef]

2. Saboori, B.; Bidgoli, A.M.; Saboori, B. Multiobjective Optimization in Repeating Sun-Synchronous Orbits Design for RemoteSensing Satellites. J. Aerosp. Eng. 2014, 27, 04014027. [CrossRef]

3. Li, D.; Wang, M.; Jiang, J. China's high-resolution optical remote sensing satellites and their mapping applications. Geo-Spat. Inf. Sci. 2020. [CrossRef]

4. Wang, M.; Cheng, Y.; Chang, X.; Jin, S.; Zhu, Y. On-orbit geometric calibration and geometric quality assessment for the high-resolution geostationary optical satellite GaoFen4. ISPRS J. Photogramm. Remote Sens. 2017, 125, 63-77. [CrossRef]

5. Wang, M.; Cheng, Y.; Tian, Y.; He, L.; Wang, Y. A New On-Orbit Geometric Self-Calibration Approach for the High-Resolution Geostationary Optical Satellite GaoFen4. IEEE J. Sel. Top. Appl. Earth Obs. Remote Sens. 2018, 11, 1670-1683. [CrossRef] 
6. Yang, B.; Pi, Y.; Li, X.; Wang, M. Relative Geometric Refinement of Patch Images Without Use of Ground Control Points for the Geostationary Optical Satellite GaoFen4. IEEE Trans. Geosci. Remote Sens. 2018, 56, 474-484. [CrossRef]

7. Toutin, T. Review article: Geometric processing of remote sensing images: Models, algorithms and methods. Int. J. Remote Sens. 2004, 25, 1893-1924. [CrossRef]

8. Cai, L.; Bu, J.; Tang, D.; Zhou, M.; Yao, R.; Huang, S. Geosynchronous Satellite GF-4 Observations of Chlorophyll-a Distribution Details in the Bohai Sea, China. Sensors 2020, 20, 5471. [CrossRef]

9. Chen, Y.; Sun, K.; Li, D.; Bai, T.; Huang, C. Radiometric Cross-Calibration of GF-4 PMS Sensor Based on Assimilation of Landsat-8 OLI Images. Remote Sens. 2017, 9, 811. [CrossRef]

10. Wei, Y.; Zhang, Z.; Mu, B.; Li, Y.; Wang, Q.; Liu, R. Geolocation Accuracy Evaluation of GF-4 Geostationary High-Resolution Optical Images over Coastal Zones and Offshore Areas. J. Coast. Res. 2020, 102, 326-333. [CrossRef]

11. Han, L.; Gao, K.; Dou, Z.; Zhu, Z.; Wang, H.; Fu, X. On-Orbit MTF Estimation for GF-4 Satellite Using Spatial Multisampling on a New Target. IEEE Geosci. Remote Sens. Lett. 2020, 17, 17-21. [CrossRef]

12. Li, P.; Sun, K.; Li, D.; Sui, H.; Zhang, Y. An Emergency Georeferencing Framework for GF-4 Imagery Based on GCP Prediction and Dynamic RPC Refinement. Remote Sens. 2017, 9, 1053. [CrossRef]

13. Yang, A.; Zhong, B.; Wu, S.; Liu, Q. Radiometric Cross-Calibration of GF-4 in Multispectral Bands. Remote Sens. 2017, 9, 232. [CrossRef]

14. Martin-Neira, M.; Oliva, R.; Corbella, I.; Torres, F.; Duffo, N.; Duran, I.; Kainulainen, J.; Closa, J.; Zurita, A.; Cabot, F.; et al. SMOS instrument performance and calibration after six years in orbit. Remote Sens. Environ. 2016, 180, 19-39. [CrossRef]

15. Wenny, B.N.; Helder, D.; Hong, J.; Leigh, L.; Thome, K.J.; Reuter, D. Pre- and Post-Launch Spatial Quality of the Landsat 8 Thermal Infrared Sensor. Remote Sens. 2015, 7, 1962-1980. [CrossRef]

16. Schwerdt, M.; Schmidt, K.; Klenk, P.; Ramon, N.T.; Rudolf, D.; Raab, S.; Weidenhaupt, K.; Reimann, J.; Zink, M. Radiometric Performance of the TerraSAR-X Mission over More Than Ten Years of Operation. Remote Sens. 2018, 10, 754. [CrossRef]

17. Jiang, B.; Liang, S.; Townshend, J.R.; Dodson, Z.M. Assessment of the Radiometric Performance of Chinese HJ-1 Satellite CCD Instruments. IEEE J. Sel. Top. Appl. Earth Obs. Remote Sens. 2013, 6, 840-850. [CrossRef]

18. Crowley, C.; Kohley, R.; Hambly, N.C.; Davidson, M.; Abreu, A.; van Leeuwen, F.; Fabricius, C.; Seabroke, G.; de Bruijne, J.H.J.; Short, A.; et al. On-orbit performance of the Gaia CCDs at L2. Astron. Astrophys. 2016, 595, 17. [CrossRef]

19. Reuter, H.I.; Nelson, A.; Jarvis, A. An evaluation of void-filling interpolation methods for SRTM data. Int. J. Geogr. Inf. Sci. 2007, 21, 983-1008. [CrossRef]

20. Gill, T.; Collett, L.; Armston, J.; Eustace, A.; Danaher, T.; Scarth, P.; Flood, N.; Phinn, S. Geometric correction and accuracy assessment of Landsat-7 ETM+ and Landsat-5 TM imagery used for vegetation cover monitoring in Queensland, Australia from 1988 to 2007. J. Spat. Sci. 2010, 55, 273-287. [CrossRef]

21. Crespi, M.; De Vendictis, L. A Procedure for High Resolution Satellite Imagery Quality Assessment. Sensors 2009, 9, 3289-3313. [CrossRef] [PubMed]

22. Ryan, R.; Baldridge, B.; Schowengerdt, R.A.; Choi, T.; Helder, D.L.; Blonski, S. IKONOS spatial resolution and image interpretability characterization. Remote Sens. Environ. 2003, 88, 37-52. [CrossRef]

23. Tao, S.; Jin, G.; Zhang, X.; Qu, H.; An, Y. Wavelet power spectrum-based autofocusing algorithm for time delayed and integration charge coupled device space camera. Appl. Opt. 2012, 51, 5216-5223. [CrossRef] [PubMed]

24. Zhang, H.; Zhang, B.; Chen, Z.; Huang, Z. Vicarious Radiometric Calibration of the Hyperspectral Imaging Microsatellites SPARK-01 and-02 over Dunhuang, China. Remote Sens. 2018, 10, 120. [CrossRef]

25. Gao, C.; Zhao, Y.; Li, C.; Ma, L.; Wang, N.; Qian, Y.; Ren, L. An Investigation of a Novel Cross-Calibration Method of FY-3C/VIRR against NPP/VIIRS in the Dunhuang Test Site. Remote Sens. 2016, 8, 77. [CrossRef]

26. Thome, K.J. Absolute radiometric calibration of Landsat 7 ETM+ using the reflectance-based method. Remote Sens. Environ. 2001, 78, 27-38. [CrossRef]

27. Vermote, E.F.; Tanre, D.; Deuze, J.L.; Herman, M.; Morcrette, J.J. Second Simulation of the Satellite Signal in the Solar Spectrum, 6S: An overview. IEEE Trans. Geosci. Remote Sens. 1997, 35, 675-686. [CrossRef]

28. Jindal, D.; Prakash, S.; Sanghvi, J.; Kartikeyan, B.; Krishna, B.G. INSAT-3D Quality Analysis System (i3dQAS). In ISPRS Technical Commission Viii Symposium; Dadhwal, V.K., Diwakar, P.G., Seshasai, M.V.R., Raju, P.L.N., Hakeem, A., Eds.; ISPRS: Hannover, Germany, 2014; Volume 40-48, pp. 257-263.

29. Ellrod, G.P.; Achutuni, R.V.; Daniels, J.M.; Prins, E.M.; Nelson, J.P. An assessment of GOES-8 imager data quality. Bull. Am. Meteorol. Soc. 1998, 79, 2509-2526. [CrossRef]

30. Jones, M.; Colombeski, N.C. Image quality control in the Meteosat Ground Processing System. Int. J. Remote Sens. 1981, 2, 331-349. [CrossRef]

31. Helder, D.; Thome, K.J.; Mishra, N.; Chander, G.; Xiong, X.; Angal, A.; Choi, T. Absolute Radiometric Calibration of Landsat Using a Pseudo Invariant Calibration Site. IEEE Trans. Geosci. Remote Sens. 2013, 51, 1360-1369. [CrossRef]

32. Bruegge, C.J.; Val, S.; Diner, D.J.; Jovanovic, V.; Gray, E.; Di Girolamo, L.; Zhao, G. Radiometric stability of the Multi-angle Imaging SpectroRadiometer (MISR) following 15 years on-orbit. In Earth Observing Systems Xix; Butler, J.J., Xiong, X., Gu, X., Eds.; International Society for Optics and Photonics: Bellingham, WA, USA, 2014; Volume 9218. 